\title{
Method for the characterization of Fresnel lens flux transfer performance
}

\author{
Juan Carlos Martínez Antón, Daniel Vázquez Moliní, Javier Muñoz de Luna, José Antonio Gómez \\ Pedrero, Antonio Álvarez Fernández-Balbuena. \\ Escuela Universitaria de Optica, UCM, C/ Arcos de Jalón 118, 28037 Madrid, SPAIN.
}

*jcmartin@,fis.ucm.es; phone 34 - 91394 6906; fax 34 - 91394 6885;

\begin{abstract}
Fresnel lenses and other faceted or micro-optic devices are increasingly used in multiple applications like solar light concentrators and illumination devices, just to name some representative. However, it seems to be a certain lack of adequate techniques for the assessment of the performance of final fabricated devices. As applications are more exigent this characterization is a must. We provide a technique to characterize the performance of Fresnel lenses, as light collection devices. The basis for the method is a configuration where a camera images the Fresnel lens aperture. The entrance pupil of the camera is situated at the focal spot or the conjugate of a simulated solar source. In this manner, detailed maps of the performance of different Fresnel lenses are obtained for different acceptance angles.
\end{abstract}

Keywords: Fresnel optics characterization, solar concentration photovoltaics

\section{INTRODUCTION}

In last decades a growing interest has been devoted to the technologies for the conversion of solar radiation into thermal or electrical energy. Among these technologies we find the high concentrating photovoltaics (HCPV) to convert solar radiation into electricity. Some efforts are made to test these HCPV devices in order to check their properties, behavior and reliability. We situate this contribution in this context, i.e. the development of instrumental tools to aid in this purpose. Many concentrating optics rely on Fresnel-like lenses. But several challenges arise in the characterization of these systems intended for concentrating photovoltaic devices.

We focus our attention on the basic design used in most HCPV technologies, i.e. a primary optics that focuses solar radiation onto a highly concentrated spot. At this spot we usually find a secondary optics with an aperture bigger than this spot ( $\sim 3-6$ times). This aperture captures the spot system misalignments and it loosens other opto-mechanical tolerances. Although the secondary optics reduces final concentration at the PV cell, it is essential to get an acceptance angle or tolerance of the system and also allows homogenizing the irradiance at the PV cell.

(a)

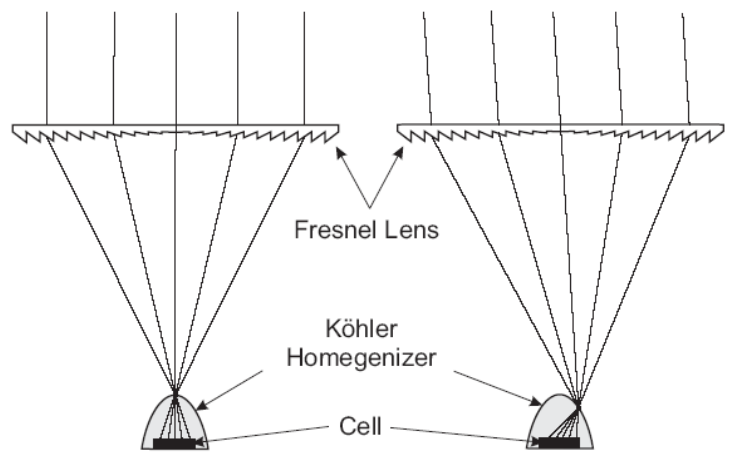

(b)

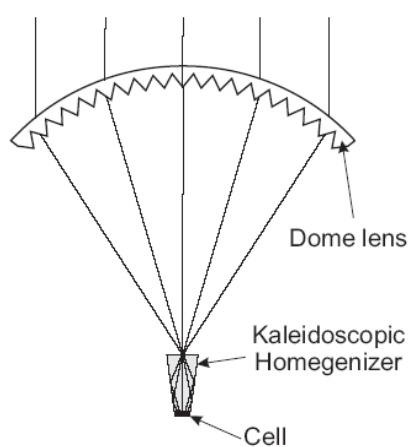

Fig. 1 Typical examples of Fresnel lens concentrators with secondary optics associated: (a) Köhler (b) prismatic

Several methods can be found in literature around the subject of characterizing the performance of solar concentrators [1-3]. Usually they analyze irradiance distribution at focal spot, sometimes masking the aperture of the concentrator to

Optical Fabrication, Testing, and Metrology IV, edited by Angela Duparré, Roland Geyl, Proc. of SPIE Vol. 8169 81690E · C 2011 SPIE · CCC code: 0277-786X/11/\$18 · doi: 10.1117/12.896870 
distinguish somehow local contributions from the primary optics to the irradiance of the focal spot [3]. Arqueros et al [4] take images of the surface of mirror solar collectors with light coming from a star. The purpose there is to provide a map of surface slopes in order to detect errors in the optics. In this work we develop a method that provides information of transfer flux performance at focal spot and simultaneously gives information on optical errors or defects at primary optics.

\section{DESCRIPTION OF THE METHOD}

\subsection{Basic setup and merit function}

An ideal Fresnel lens acting as a primary solar concentrator should focus the solar disk in its image at focal plane in paraxial approximation or to a focal spot close to this size considering the Fresnel lens as a non-imaging device. Typically a secondary optics is needed to capture optical and solar misalignments as illustrated in Fig. 1(a), but also to hold the optical tolerances of the primary concentrator. Then the secondary optics works capturing the solar spot displacements and blurring, but also homogenizing irradiance at the level of PV cell. Lenses working as Köhler illuminating components (Fig. 1(a)) or prismatic homogenizers (Fig. 1(b)) are common practice for secondary optics.

The aperture of the secondary optics determines the acceptance angle of the solar concentrator. For a circular aperture, it means a circular field of view of the concentrator optics as shown in Fig. 2(a). However, practical optics is imperfect, especially Fresnel optics, where optical tolerances are compromised by the goal of low cost production. In this sense we can see any optical primary concentrator as a sum of finite optical elements working to concentrate solar beams at the expected focus (Fig. 3). Considering the problem in reverse, from the nominal focal spot (solar disk image), in an ideal system all rays come from the sun disk. This is illustrated in Fig. 3 with discontinuous lines. In a real system, any small area of the lens ("dots" in the Figs. 2-4) works slightly different as would be expected. This situation is illustrated in Fig. 4. Every lens dot directs light to the focal spot but capturing it from different fields of view as we illustrate in Fig. 2(b).

For a primary concentrator the objective is to concentrate the light to a spot, which in the best case it should have the size of the solar disk image in paraxial approximation. We present a method to characterize performance inspired in known lens techniques for aberration estimation [5]. However it is adapted to flux transfer estimation instead of imaging or aberration performance.

(a)

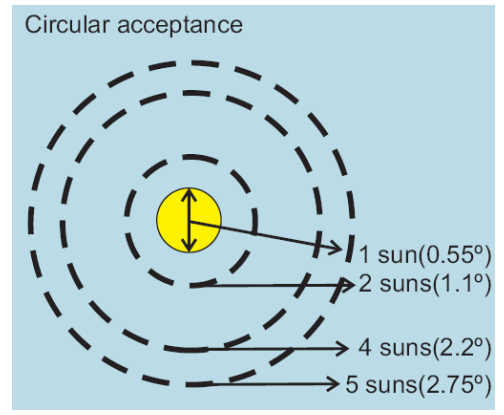

(b)

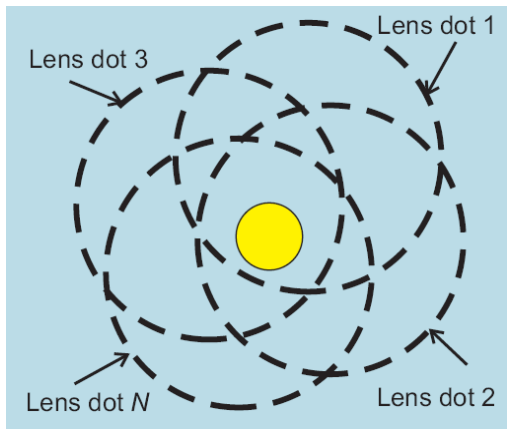

Fig. 2 Description of acceptance angles ( field of view) for a concentrator with circular aperture at the secondary optics. (a) An ideal primary concentrator provides concentric acceptance proportional to the aperture size of the secondary. (b) In an imperfect primary concentrator every lens element (dot) works slightly different (details in text).

We define the following merit function to evaluate the performance or efficiency of a concentrating lens as

$$
\eta=\frac{L_{\text {Lens }}}{L_{E L D}},
$$

where $L_{L e n s}$ is the radiance of the primary optics seen from the focal spot when concentrating a solar disk, and $L_{E L D}$ is the radiance of an Equivalent Lambertian Disk, situated at the lens position with the same radiance of the solar disk (or simulated source) and lambertian distribution, i.e. with radiance constant with angle of observation. In a measurement, the radiance of both situations is measured with an imaging camera with a finite entrance pupil size kept fixed. In a system without losses the radiance is preserved and it's a characteristic constant of the propagated beam (also known as éténdue or optical invariant). Therefore in an ideal concentrator this condition should be fulfilled and $\eta=1$. In a real lens, 
reflection losses and aberrations would lower this value. Fresnel lenses have even more losses due to ridge scattering and diffraction and are prone to more aberration and surface errors.

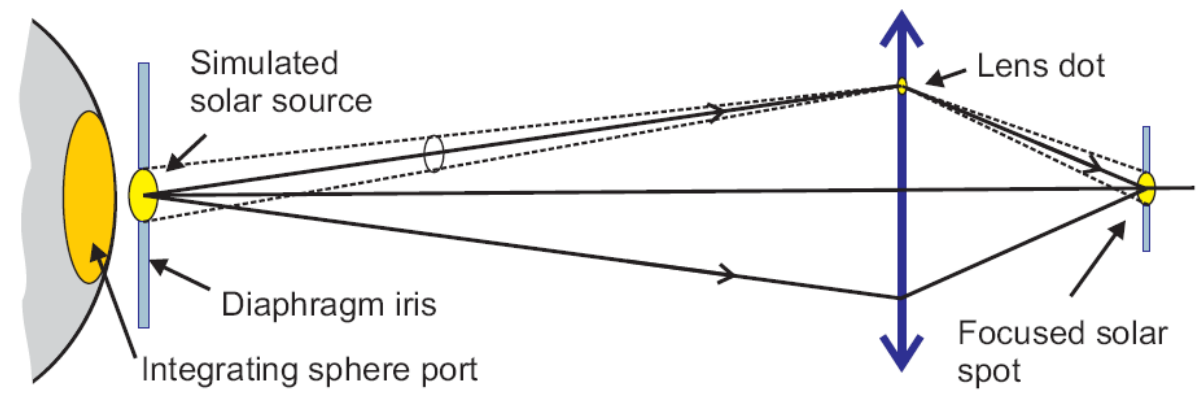

Fig. 3 Optical setup for measurement under ideal imaging performance.

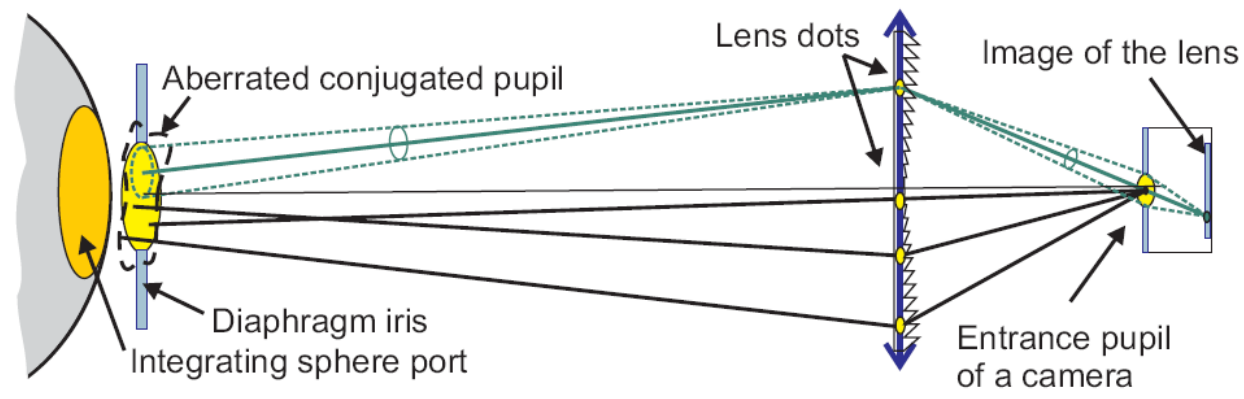

Fig. 4 Description of the setup with an imperfect lens. Aberrations are considered using the optical reversibility principle.

\subsection{Procedure}

Any imaging device (a CMOS camera in our case) is a sort of radiance meter. The captured images provide a map of the radiance of the object point by point (or a proportional value). We situate the entrance pupil (EP) of a camera in the focal spot of a Fresnel lens to get an image of the lens aperture working as a light collection device. The diameter of EP is 1.4 $\mathrm{mm}$ in our experiments. As a simulated solar disk we use an exit port of an integrating sphere with variable size, located far away from the lens to approach infinity (at $0.4 \mathrm{dp}$ in our measurements). At this position we take a picture of the lens providing a relative map of performance. This image gives $L_{\text {lens }}$. As equivalent lambertian disk, we use the mentioned integrating sphere, where we take a picture inside overfilling the field of view of the camera. This serves as the reference image, giving $L_{E L D}$ value.

In our experiments, the integrating sphere is internally illuminated with 3 light emitting diodes centered at wavelengths of $\lambda_{\mathrm{B}}=455 \mathrm{~nm}, \lambda_{\mathrm{B}}=510 \mathrm{~nm}$ and $\lambda_{\mathrm{B}}=630 \mathrm{~nm}$. The camera takes RGB pictures that together with the tri-chromatic illumination allows for a good color contrast and chromatic aberration discrimination. To evaluate the overall performance or efficiency we sum and averaged the three RGB channels of the camera device with the same weight. In a real solar irradiation and depending on the spectral band of detection, the effect of chromatic aberration is expected to be worse than the results provided by our solar disk simulator of limited spectral bandwidth.

The parameter $\eta$ has been measured for different light source sizes (diameter of the diaphragm iris of Fig. 3) as a way to know the performance for different fields of view. To take this size in consideration, we conveniently define the normalized acceptance $A^{*}$ :

$$
A^{*}=\frac{\omega_{S D}}{\omega_{E P}},
$$

where $\omega_{E P}$ and $\omega_{S D}$ are respectively the subtended angles of the entrance pupil of the camera and the simulated solar disk (iris diaphragm) seen from the lens at its optical axis. A normalized acceptance of $A^{*}=1$, means that the simulated solar disk paraxial image fits exactly in the entrance pupil (in an ideal system). According to eq. (1) for $A^{*}>1$ the efficiency $\eta$ should be just 1 and $\eta$ below 1 for $A^{*}<1$. Notice that the radiance measured by the camera is for specific $\omega_{E P}$. 


\section{RESULTS}

Under the introduced configuration we analyze the performance of several Fresnel Lenses (4 types described in Fig.5 caption). Two series of measurements are given. First we analyze the chromatic aberration by displacing the camera along the optical axis of the lenses near the best focus (see Fig.5) for a normalized acceptance of $A^{*}=1$. While doing it, it is possible to appreciate fabrication defects and poor performance.

(A)

\section{Blue focus $\left(0.96 \cdot f^{\prime}\right)$}
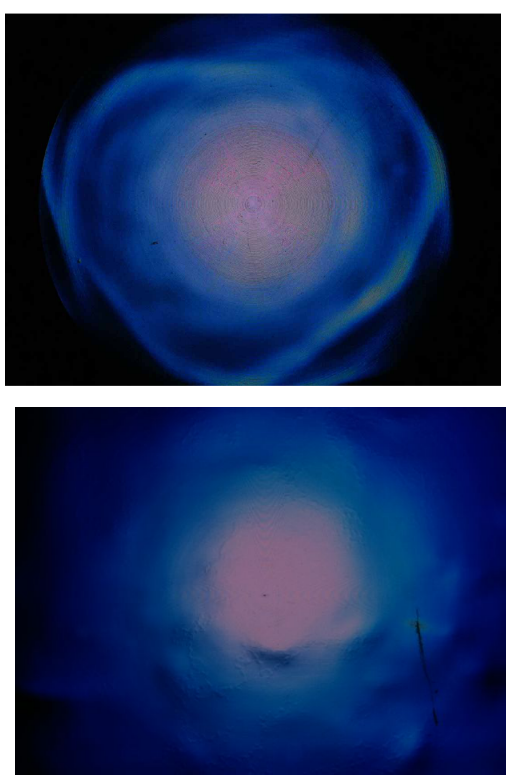

(B)

(C)
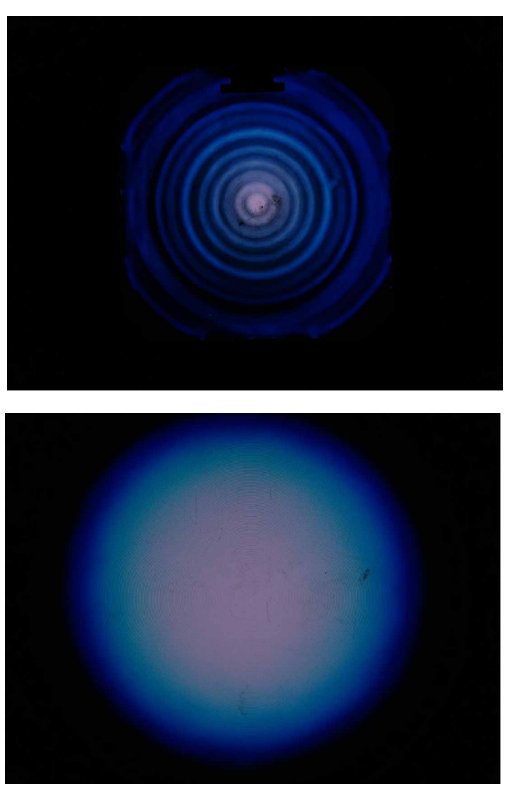

Best focus $\left(f^{\prime}\right)$
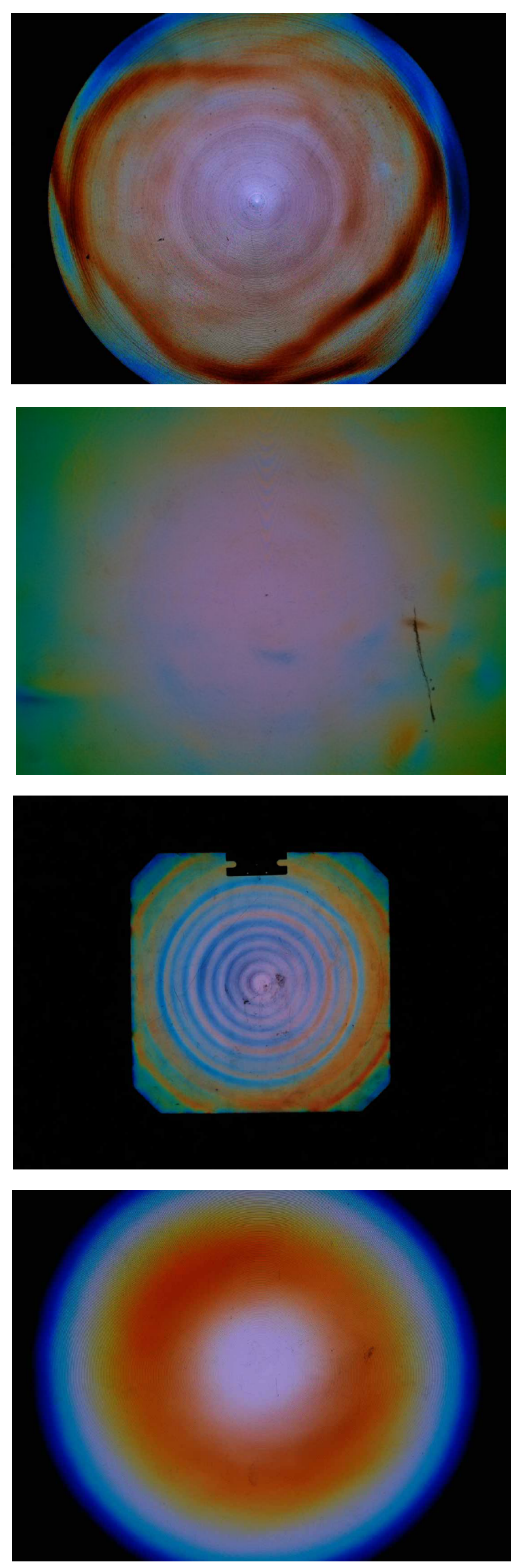

Red focus $\left(1.04 \cdot f^{\prime}\right)$
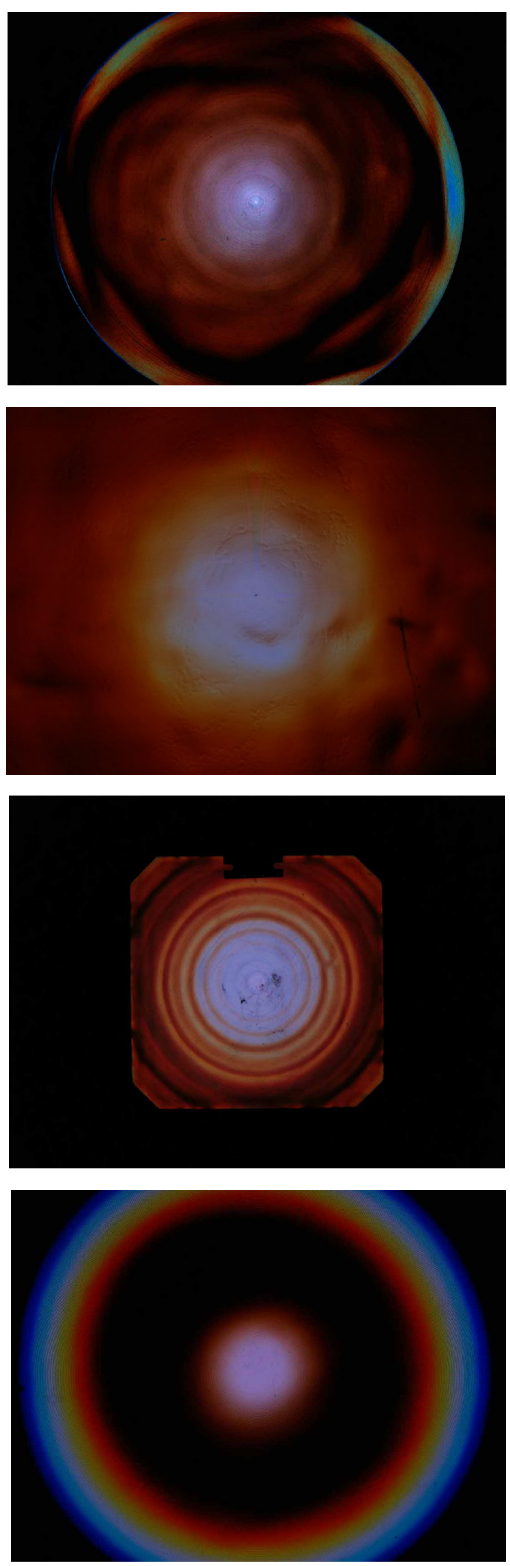

Fig. 5 Lens performance maps related to chromatic aberration (left to right). Frame FOV $= \pm 25^{\circ} \mathrm{x} \pm 20^{\circ}$. Left column: EP at a distance of the lens of $0.96 \cdot f^{\prime}$, center column: $\mathrm{EP}$ at best focus $\left(f^{\prime}\right)$, right column: $\mathrm{EP}$ at $1.04 \cdot f^{\prime}$. (A) $\mathrm{L}_{\mathrm{A}}: \mathrm{D}=100 \mathrm{~mm}$, $\mathrm{f}^{\prime}=120 \mathrm{~mm}$, pitch $=0.2 \mathrm{~mm},(\mathrm{~B}) \mathrm{L}_{\mathrm{B}}: \mathrm{D}=230 \times 230 \mathrm{~mm}, \mathrm{f}^{\prime}=200 \mathrm{~mm}$, pitch=0.2 mm, (C) $\mathrm{L}_{\mathrm{C}}: \mathrm{D}=280 \times 280 \mathrm{~mm}, \mathrm{f}^{\prime}=450 \mathrm{~mm}$, pitch $=0.2 \mathrm{~mm}$, (D) $\mathrm{L}_{\mathrm{D}}: \mathrm{D}=230 \times 230 \mathrm{~mm}, \mathrm{f}^{\prime}=200 \mathrm{~mm}$, pitch $=0.5 \mathrm{~mm}$. (Color on line) 

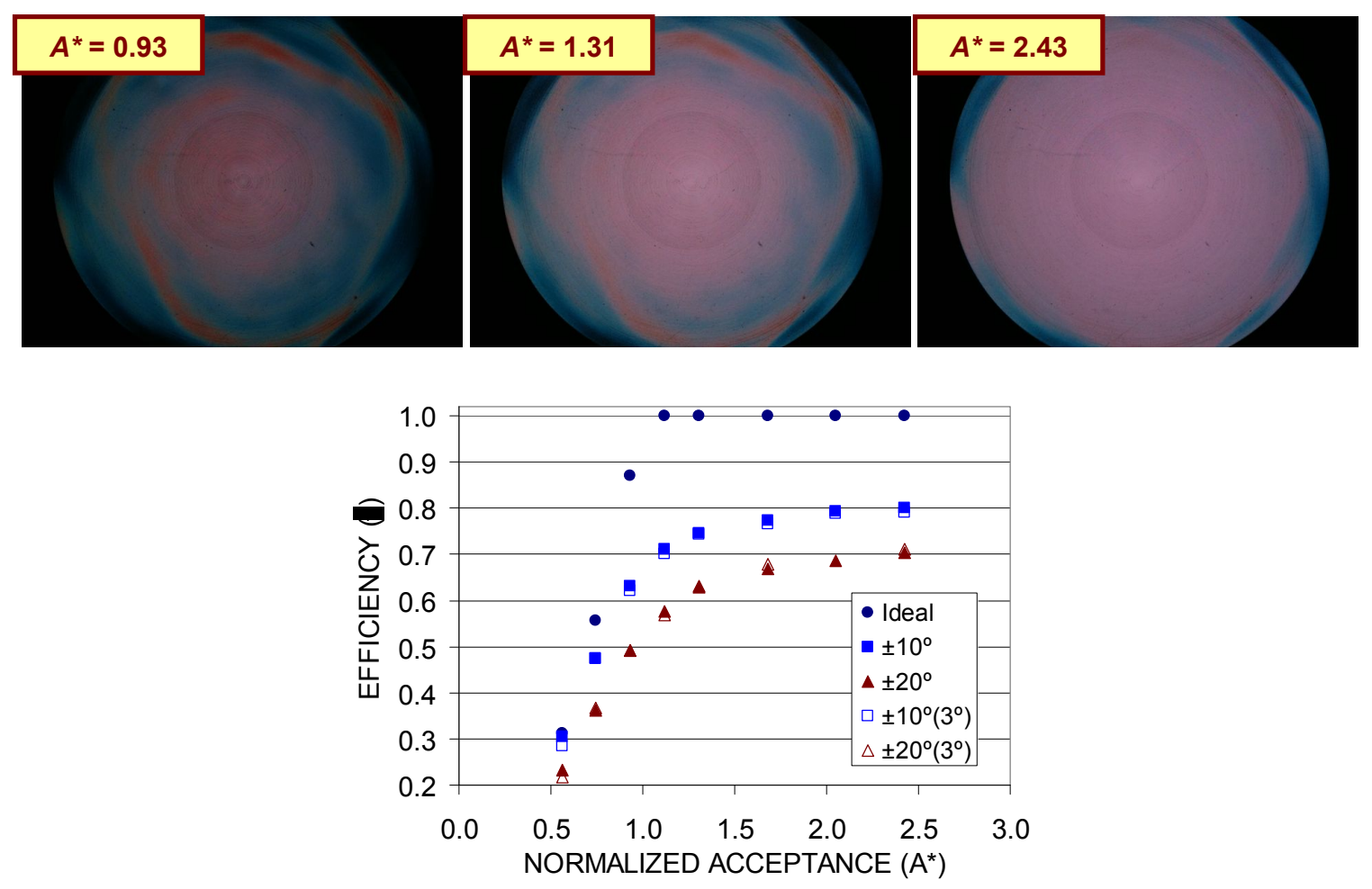

Fig. 6 Optical performance of $\mathrm{L}_{\mathrm{A}}$ versus normalized acceptance for different FOV. Above: specific images of the series $\left(0^{\circ}\right)$.
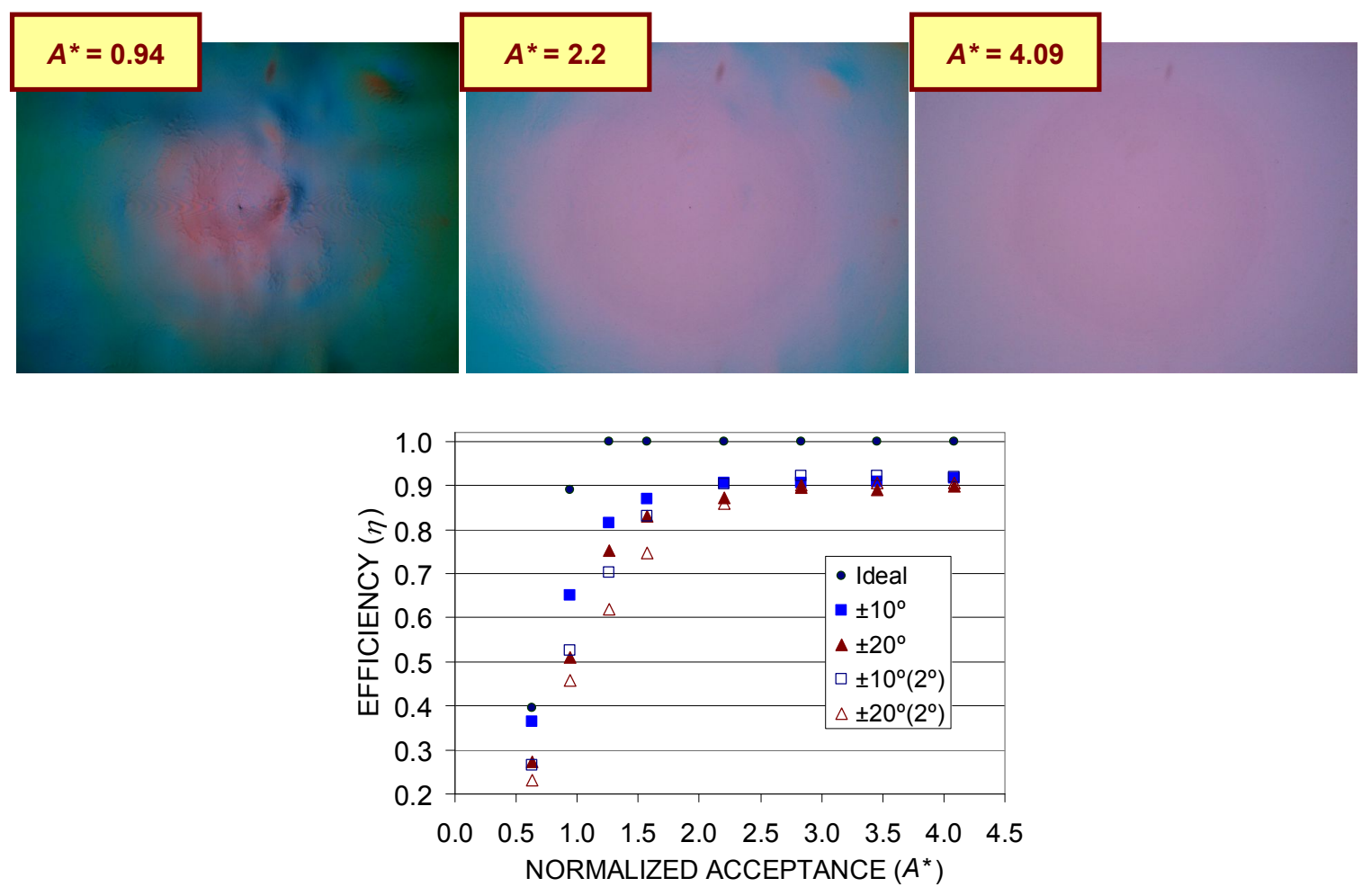

Fig. 7 Optical performance of $L_{B}$ versus normalized acceptance for different FOV. Above: specific images of the series $\left(0^{\circ}\right)$ 

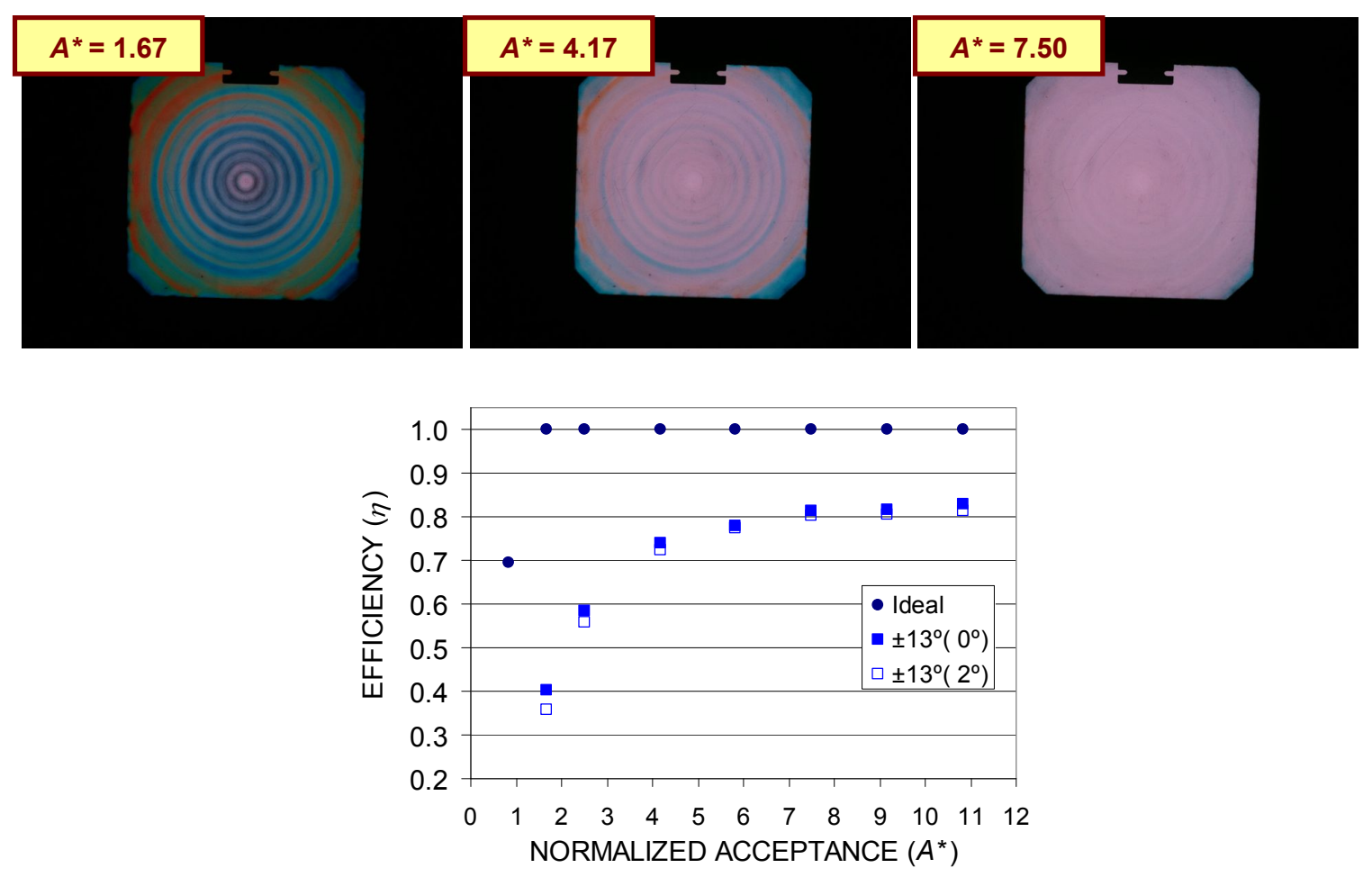

Fig. 8 Optical performance of $\mathrm{L}_{\mathrm{C}}$ versus normalized acceptance for different FOV. Above: specific images of the series $\left(0^{\circ}\right)$
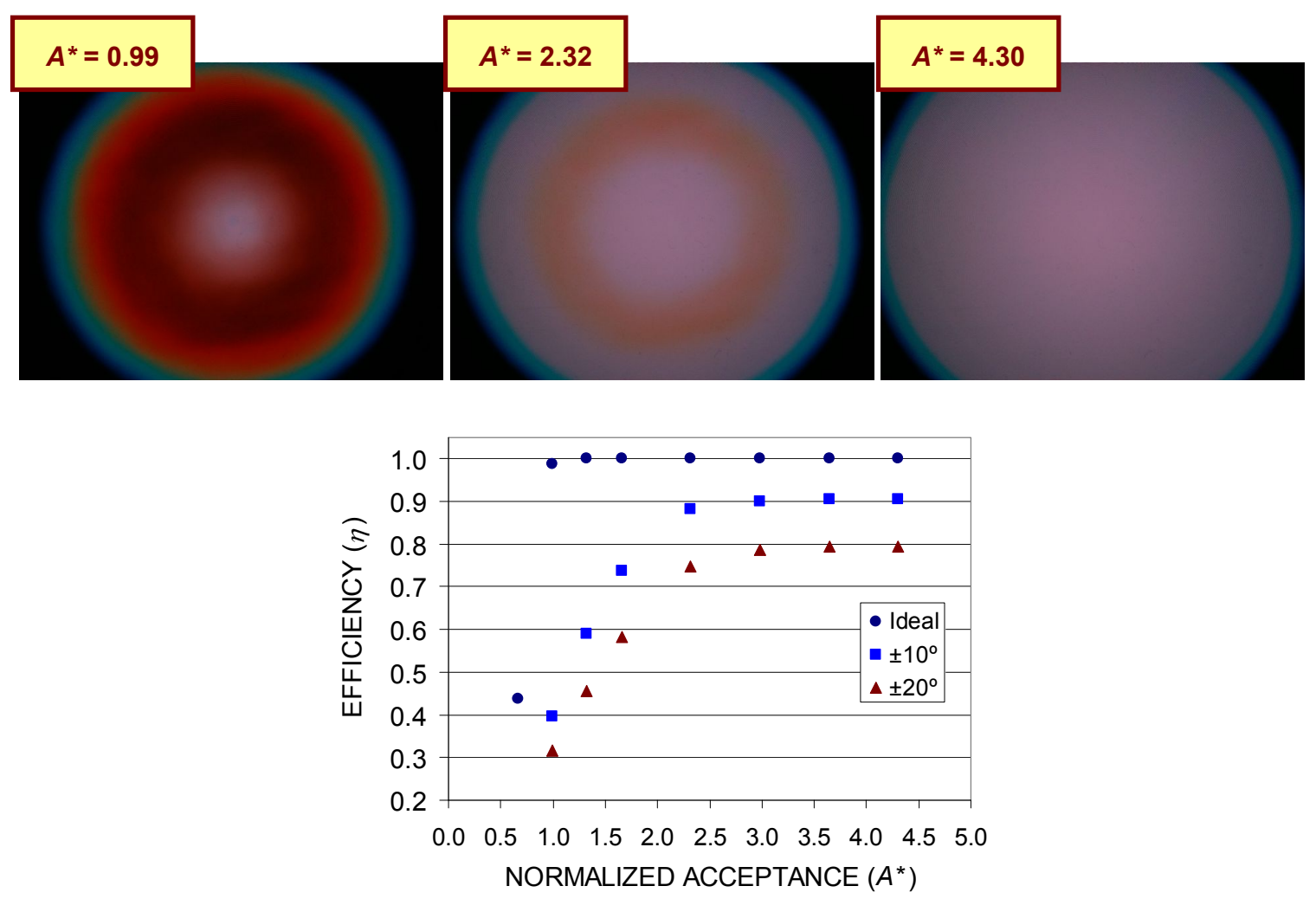

Fig. 9 Optical performance of $L_{D}$ versus normalized acceptance for different FOV. Above: specific images of the series $\left(0^{\circ}\right)$. 
Secondly, the transfer flux performance was measured following the mentioned procedure by varying simulated solar disk size and therefore the normalized acceptance $\left(A^{*}\right)$. We obtain the averaged efficiency for different field of views of the observed lens (lens aperture), typically at $\pm 10^{\circ}$ and $\pm 20^{\circ}$ (see Fig. 6-Fig. 9) and for a solar disk misalignment of $2^{\circ}$ or $3^{\circ}$. Lenses A and B are "best from lenses" i.e. with facets oriented to simulated solar disk, and lenses C and D follow a more classical design in solar applications, with flat side facing the solar simulated disk. In these two last cases it is appreciated a higher sensitivity to solar misalignment (Fig. 7).

\section{Conclusion}

A method of characterization of flux transfer in lenses specially adapted to HCPV technologies has been developed. It allows a quick and direct estimation of overall optical efficiency while simultaneously provides a map of the efficiency of the lens point by point, informing in detail of lens errors and defects. From the results of the studied lenses we can extract the conclusion that flux performance decay significantly by increasing the working aperture of a lens. Not only chromatic aberration is an issue, small surface angle errors in the lens have a bigger effect than in paraxial working area.

\section{REFERENCES}

[1] Chemisana, D., Vossier, A., Pujol, L., Perona, A., Dollet A., "Characterization of Fresnel lens optical performances using an opal diffuser", Energy Conversion and Management 52, 658-663 (2011)

[2] Andersen, M., Rubin, M., Scartezzini, J.L., "Comparison between ray-tracing simulations and bi-directional transmission measurements on prismatic glazing", Sol Energy 74, 157-73 (2003)

[3] Sansoni, P., Francini, F., Fontani, D. "Optical characterization of solar concentrator", Optics and Lasers in Engineering 45, 351-359 (2007)

[4] Arqueros, F., Jimenez, A., Valverde, A., "A novel procedure for the optical characterization of solar concentrators", Solar Energy 75, 135-142 (2003)

[5] Ojeda-Castañeda, J., "Foucault, wire and phase modulation tests" in Optical Shop Testing, $3^{\text {rd }}$ edition, Ed. Daniel Malacara, Wiley-Interscience, (2007). 